\title{
RBP- $\mathrm{J} \kappa$ repression activity is mediated by a co-repressor and antagonized by the Epstein-Barr virus transcription factor EBNA2
}

\author{
L. Waltzer, P. Y. Bourillot, A. Sergeant and E. Manet ${ }^{\star}$ \\ Unit de Virologie Humaine, ENS-INSERM U412, Ecole Normale Supérieure de Lyon, 46 Allée d'Italie, 69364 \\ Lyon Cedex 07, France
}

Received October 10, 1995; Revised and Accepted November 13, 1995

\begin{abstract}
The Epstein-Barr virus (EBV) protein EBNA2 is a transcriptional activator that can be targeted to its DNA responsive elements by direct interaction with the cellular protein RBP-JK. RBP-JK is a ubiquitous factor, highly conserved between man, mouse and Drosophila, whose function in mammalian cells is largely unknown. Here we provide evidence that RBP-JK is a transcriptional repressor and, more importantly, that RBP-JK repression is mediated by a co-repressor. The function of the co-repressor could be counterbalanced by making a fusion protein (RBPVP16) between RBP-JK and the VP16 activation domain. This RBP-VP16-mediated activation could be strongly increased by an EBNA2 protein deprived of its activation domain, but not by an EBNA2 protein incapable of making physical contact with RBP-JK. Our results suggest that EBNA2 activates transcription by both interfering with the function of a co-repressor recruited by RBP-JK and providing an activation domain.
\end{abstract}

\section{INTRODUCTION}

Epstein-Barr virus (EBV) is a widespread human herpes virus associated with several human malignancies, including Burkitt's lymphoma, nasopharyngeal carcinoma, Hodgkin's disease and various lymphomas in immunodepressed patients (1). In vitro infection of B lymphocytes by EBV and culture of lymphocytes from seropositive individuals give rise to immortalized cell lines. In such immortalized B cells EBV persists mainly as an episome, from which a limited set of viral genes is expressed that characterizes EBV latency (type III latency). This EBV-induced immortalization is absolutely dependent on the EBV nuclear protein EBNA2 $(2,3)$. EBNA2 is necessary not only for the initiation (4), but also for the maintenance of immortalization (5). EBNA2 has been shown to act as a transcriptional activator. In particular, it up-regulates expression of the cellular genes $C D 21, C D 23$ and c- $f g r(6-9)$ and the EBV genes $L M P$ and TPI (10-13). Moreover, transcription of EBNA genes initiated at the $\mathrm{Cp}$ promoter is also activated by EBNA2 (14-16). The capacity of EBNA2 to activate transcription has been directly linked to its role in immortalization (17). Recently important advances have been made in the understanding of the mechanisms by which EBNA2 activates transcription: EBNA2 does not interact directly with DNA, but appears to be recruited to promoters by the cellular DNA binding protein RBP-JK (also called KBF2 and CBF1) (18-24).

The RBP-JK protein was first characterized in the mouse $(25,26)$ and then the corresponding cDNAs were cloned from mouse, man and Drosophila (26-29). The DNA recognition sequence has been precisely characterized and contains the core sequence GTGGGAA, which is present in all known EBNA2-responsive promoters (30). In addition to being a DNA binding intermediary protein for EBNA2, other evidence suggest that RBP-JK could be a more general transcriptional regulator. Indeed, RBP-JK has been identified in Drosophila as Suppressor of Hairless [ $\mathrm{Su}(\mathrm{H})]$, a nuclear protein involved in development of the peripheral nervous system (31-33). In S2 Drosophila cells $\mathrm{Su}(\mathrm{H})$ or its human homologue RBP-JK can act as a transcriptional activator in a transient transfection assay (28). In mammalian cells RBP-JK has been described as a transcriptional repressor of the adenovirus $p I X$ gene, in which a binding site for RBP-JK is found adjacent to the TATA box (34). Furthermore, RBP-JK fused to the DNA binding domain of Gal4 is able to down-regulate transcription from the herpes simplex virus thymidine kinase $(T K)$ promoter linked to five Gal4 binding sites (35). The authors of this study suggest that RBP-JK contains a repression domain and that EBNA2 bound to RBP-JK not only provides RBP-JK with an activation domain, but also impairs RBP-JK-induced repression, either by masking the repression domain in RBP-JK or by interfering with its function. However, this model is based on studies with Gal4-RBP-JK and awaits confirmation using RBP-JK bound to its cognate binding sites. Furthermore, nothing is known of the mechanisms of repression by RBP-JK and more specifically whether it influences transcription directly or requires a co-effector.

In the present study we mimic the recruitment of an activation domain by RBP-JK without concomitant masking of the RBP-JK repression domain. Moreover, we demonstrate that RBP-JK repression is mediated by a co-repressor and that although this repression is impaired by EBNA2, this latter effect does not appear to involve direct contact between EBNA2 and the co-repressor.

${ }^{*}$ To whom correspondence should be addressed 


\section{MATERIALS AND METHODS}

\section{Reporter plasmids}

The reporter plasmids pTK-CAT-Cp1x, pTK-CAT-Cp2x and pTK-CAT-Cp4x were generated by inserting one, two and four copies respectively of a Xhol-Sall double-stranded oligonucleotide containing the RBP-JK binding site of the Cp promoter 5'-TCGAGGTGTAAACACGCCGTGGGAAAAAATTTTG-3'

3'-CCACATTTGTGCGGCACCCTTTTTTAAATACACT-5' into the XhoI site of plasmid pBLCAT2. The plasmids pTK-CATCpM1x, pTK-CAT-CpM2x and pTK-CAT-CpM4x were generated by inserting one, two and four copies respectively of a XhoI-Sall double-stranded oligonucleotide containing a mutated counterpart of the RBP-JK binding site

5'-TCGAGGTGTAAACACGAATTCGGAAAAAATATG-3'

3'-CCACATTTGTGCTTAAGCCTTTTTTAAATCAGCT-5' into the XhoI site of plasmid pBLCAT2. pTATA-CAT-Cp4x was derived from pTK-CAT-Cp4x by removing an AvaII-BamHI DNA fragment from the $T K$ promoter and religating the plasmid after blunting the extremities with the DNA polymerase I large fragment (Klenow).

\section{Expression vectors}

Plasmid pSG5Flag was derived from pSG5 (Stratagene) by insertion between the EcoRI and BamHI sites of a doublestranded oligonucleotide containing the sequence encoding the Flag peptide (IBI Flag system, Kodak). The full-length human RBP3 cDNA was subcloned from pT7ßlinkRBP3 (28) into the BamHI site of the pSG5Flag vector to generate pSG5Flag-RBP3. Site-directed mutagenesis into pSG5Flag-RBP3 was carried out with the Clontech Transformer ${ }^{\mathrm{TM}}$ site-directed mutagenesis kit. The mutagenesis was designed so as to specifically change two amino acids and to introduce a HindIII restriction site. Sequences of the synthetic oligonucleotides used to generate the RBP3 mutants KR50SL, KK68SL, KR121SL, KK156SL, RL178GI, DD212SL, EE219GS, EEF219AAA, HK269SL, MV282SW, CP299SL, WT314SL, YT324SL, GG349SW, WF370SL, YT430SL and NS473SL were as follows.

$\begin{array}{ll}\text { KR50SL } & \text { GGAAATGAAAGCTTGTTTTTTGCCC } \\ \text { KK68SL } & \text { CGGATGGAAAAGCTTAAAAGAACAAATGG } \\ \text { KR121SL } & \text { CTGACTCAGAAAGCTTAAAGCACTTCA } \\ \text { KK156SL } & \text { ACCTTCCAAAAGCTTGCAGTCATTG } \\ \text { RL178GI } & \text { GGCTCTGTTTAATGGAATTCGATCCCAG } \\ \text { DD212SL } & \text { TTCATCTCTTAAGCTTAGATGAATCAGAAGG } \\ \text { EE219GS } & \text { GAATCAGAAGGAGGATCCTTCACAGTCCG } \\ \text { EEF219AAA } & \text { GAATCAGAAGGAGCAGCAGCCACAGTCCGAG } \\ \text { HK269SL } & \text { GTGTCACAACTAAGCTTATGTGCATTTACC } \\ \text { MY282SW } & \text { ACAGAAAGAAGCTTTTGTGCCTTT } \\ \text { CP299SL } & \text { GCCACTCCAAGCTTAAAAGAACCAA } \\ \text { WT314SL } & \text { ATGGCGCTTCAAGCTTAATCATTAGCAC } \\ \text { YT324SL } & \text { GATAAGGCAGAAAGCTTATTTATGAGGG } \\ \text { GG349SW } & \text { CTTCAGTTGAAAAGCTTTGGGGACGTAG } \\ \text { WF370SL } & \text { ATTTACGAGTAAGCTTAGGGGATGTAG } \\ \text { YT430SL } & \text { CTTACCTTTACAAGCTTACCAGAACCAGG } \\ \text { NS473SL } & \text { GCCAGCACAAGCTTAACCAGTGTC. }\end{array}$

The double mutant RBP KR50SL/EEF219AAA was generated by simultaneously using the two oligonucleotides KR50SL and EEF219AAA for site-directed mutagenesis into pSG5FlagRBP3.
The transcriptional activation domain of VP16 (amino acids 402-479) was isolated from pMC1 (36) by PCR using oligonucleotides 5'-GAATTCCAAAGCTTGGCCCCCCCGACCGATG-3' and 5'-CTCGGATCCTACCCACCGTACTCG-3'. The PCR products were digested with HindIII and BamHI and subcloned into plasmid pSG5Flag-RBP NS473SL, between the HindIII and BglII sites (the HindIII site is contained in the mutagenic NS473SL oligonucleotide), to generate pSG5Flag-RBP-VP16.

The pSG5-EBNA2 mutants were obtained via linker insertion mutagenesis. pSG5-EBNA2 (20) was partially digested with NlaIV or HaeIII in the presence of ethidium bromide, the extremities were blunted using the Klenow DNA polymerase and then ligated to a 12 mer oligonucleotide HindIII linker (Biolabs). Deletion mutants were generated by cutting different linker insertion mutants with HindIII and at the unique EcoRI site present in the vector and then religating the appropriate DNA fragments to generate in-frame deletions. Plasmids pSG5-EBNA2 $\triangle 321-323$ and pSG5-EBNA2 $\triangle 437-477$ were generated in this way.

\section{Synthesis of in vitro-translated proteins and EMSA}

In vitro-translated proteins were all synthesized in a reticulocyte lysate coupled transcription/translation system (Promega). Electrophoretic mobility shift assays (EMSA) were performed by incubating $5 \times 10^{4}$ c.p.m. 5'-end-labeled double-stranded templates with in vitro-translated proteins for $30 \mathrm{~min}$ at room temperature in $10 \mathrm{mM}$ HEPES, pH 7.9, $50 \mathrm{mM} \mathrm{KCl}, 0.5 \mathrm{mM}$ $\mathrm{MgCl}_{2}, 1 \mathrm{mM}$ EDTA, $0.5 \mathrm{mM}$ dithiothreitol (DTT), $8 \%$ glycerol and $1 \mu \mathrm{g}$ poly $(\mathrm{dI}-\mathrm{dC})$ in a final volume of $20 \mu \mathrm{l}$. Reticulocyte lysate (RRL) was added to the required reactions in order to keep the overall amount of RRL constant. After incubation the mixture was loaded onto a $4.5 \%$ (w/v) polyacrylamide gel (29:1 cross-linked) with $0.2 \times \mathrm{TBE}$ and run at room temperature at $10 \mathrm{~V} / \mathrm{cm}$ for $3 \mathrm{~h}$. The protein-DNA complexes were visualized by autoradiography.

\section{Transfections}

The plasmids used for transfection were prepared by the alkaline lysis method and purified through two $\mathrm{CsCl}$ gradients. The DNAs were in the same topological state, as assayed by agarose gel electrophoresis. HeLa cells were grown in Dulbecco's modified Eagle's medium (Gibco) supplemented with $10 \%(v / v)$ fetal calf serum and were seeded at $5 \times 10^{5}$ cells $/ 100 \mathrm{~mm}$ Petri dish $8 \mathrm{~h}$ prior to transfection. Transfections were performed by the calcium phosphate precipitation method. Cells were mixed with the appropriate DNAs; typically $15 \mu \mathrm{g}$ DNA was used, which included the expressing vector and plasmids carrying the reporter genes. The pSG5 expression vector was added when required, in order to keep the amount of SV40 promoter constant. Transfected cells were washed and collected $48 \mathrm{~h}$ after transfection. Transfection experiments were performed at least three times, with reproducible results.

\section{CAT-ELISA}

CAT-ELISA were performed using the Boehringer Mannheim CAT-ELISA kit following the manufacturer's instructions. After transfection cells were lysed in $1 \mathrm{ml}$ lysis buffer and the amount of CAT protein produced was calculated for the totality of the protein extract. 
A

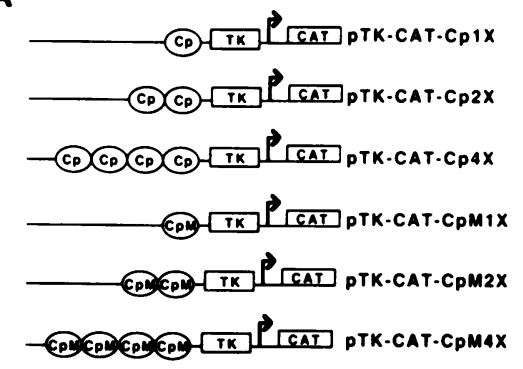

$\mathbf{B}$

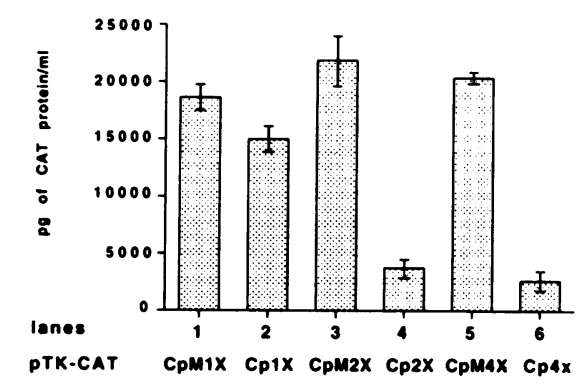

C

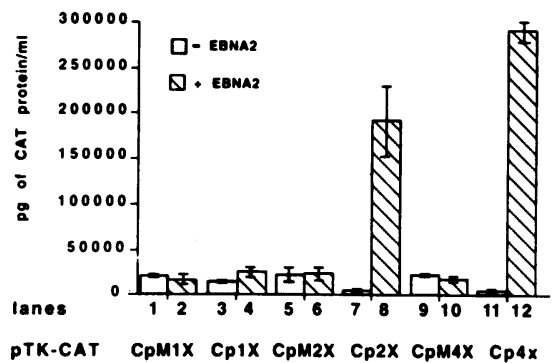

Figure 1. RBP-JK binding sites mediate repression of $T K$ promoter activity and transactivation by EBNA2. (A) Schematic representation of the reporter plasmids. (B) The various reporter plasmids $(10 \mu \mathrm{g})$ were transfected into $\mathrm{HeLa}$ cells. The promoter activity was assayed by quantifying the amount of CAT protein expressed by CAT-ELISA. (C) The various reporter plasmids $(10 \mu \mathrm{g})$ were co-transfected in HeLa cells with $1 \mu \mathrm{g}$ of either the pSG5 (dotted columns) or pSG5-EBNA2 (hatched columns) expression vector.

\section{RESULTS}

Endogenous RBP-JK represses transcription from a $T K$ promoter containing binding sites for RBP-JK

In order to study both the role of RBP-JK in transcriptional regulation and the mechanisms of activation by EBNA2 we used a series of plasmids (see Fig. 1A) which contain either one, two or four copies of a RBP-JK binding site upstream of the herpes simplex virus $T K$ promoter linked to the $C A T$ gene. We have also generated an equivalent series of constructions in which the RBP-JK binding sites are mutated so that they no longer bind RBP-JK.

As shown in Figure 1B, upon transfection in HeLa cells comparable amounts of CAT protein were expressed from the reporter plasmids containing one, two or four mutated RBP-JK binding sites (lanes 1, 3 and 5), thus demonstrating that the insertion of DNA sequences did not affect $T K$ promoter activity. However, reduced amounts of CAT protein were expressed from the constructs containing one, two and four RBP-JK binding sites as compared with their mutated counterparts (compare lanes 2, 4 and 6 with lanes 1, 3 and 5). Similar results were obtained with EBV-negative DG75 B cells (data not shown). It is noteworthy that although one RBP-JK binding site was sufficient to mediate repression (compare lanes 1 and 2), two or four binding sites mediated a synergistic repression (compare lanes 4 and 6 with lane 2). These results suggest that in HeLa cells an endogenous factor is able to repress transcription through RBP-JK binding sites.

The most likely candidate for this endogenous factor is RBP-JK itself, as it is the only HeLa cell factor that has been shown to specifically bind to the core sequence GTGGGAA (21; our own unpublished data). Moreover, EBNA2 was able to activate transcription from the $T K$ promoter carrying RBP-JK binding sites (Fig. 1C, compare lanes 4, 8 and 12 with lanes 3,7 and 11), but not from the TK promoter carrying mutated RBP-JK binding sites (Fig. 1C, compare lanes 2, 6 and 10 with lanes 1, 5 and 9). This is another indication that endogenous RBP-JK binds the reporter plasmids. It should be stressed that although one RBP-JK binding site was sufficient to mediate EBNA2 activation (Fig. 1C, compare lanes 4 and 3), two or four binding sites mediated a synergistic EBNA2 activation (Fig. 1C, compare lanes 8 and 12 with lane 4).

Since RBP-JK appears to be a repressor, RBP-JK-mediated EBNA2 transcriptional activation could be due to several different mechanisms. For example, the EBNA2 activation domain could counterbalance the repression mediated by RBP-JK. Alternatively, by making physical contact with RBP-JK, EBNA2 could interfere with RBP-JK repression mediated either directly or by a co-repressor. Finally, as suggested by the strong destabilizing effect of EBNA2 on the RBP-JK-DNA complexes formed in vitro (20), EBNA2 could remove RBP-JK from its target promoter.

\section{RBP-JK provided with the VP16 activation domain becomes an activator}

First we tested whether providing RBP-JK with an activation domain would be sufficient to counterbalance repression. For this purpose the sequence coding for the acidic activation domain of the herpes simplex virus VP16 transcriptional activator was fused to the C-terminus of the RBP3 coding sequence (RBP3 being one of the known RBP-JK isoforms; 28) (Fig. 2A). This protein, called RBP-VP16, bound DNA as efficiently as RBP3 (Fig. 2B, lane 2 and 6) in an EMSA. We next evaluated whether RBP-VP16 could activate transcription. As shown in Figure 3, RBP-VP16 activated transcription when RBP-JK binding sites were present upstream of the $T K$ promoter (compare lanes 4, 6, 8, 10 and 12 with lane 2), whereas mutated RBP-JK binding sites did not confer responsiveness to RBP-VP16 (lanes 1, 3, 5, 7, 9 and 11). Thus our results clearly show that RBP-JK provided in cis with an activation domain functions as an activator. As a consequence, the VP16 activation domain added in cis to RBP-JK counterbalanced RBP-JK-mediated repression. Moreover, masking of the RBP-JK putative repression domain by EBNA2 is not absolutely required to obtain RBP-VP16-mediated activation. Similar results were obtained using RBP3 fused to the EBNA2 activation domain (data not shown). 
A
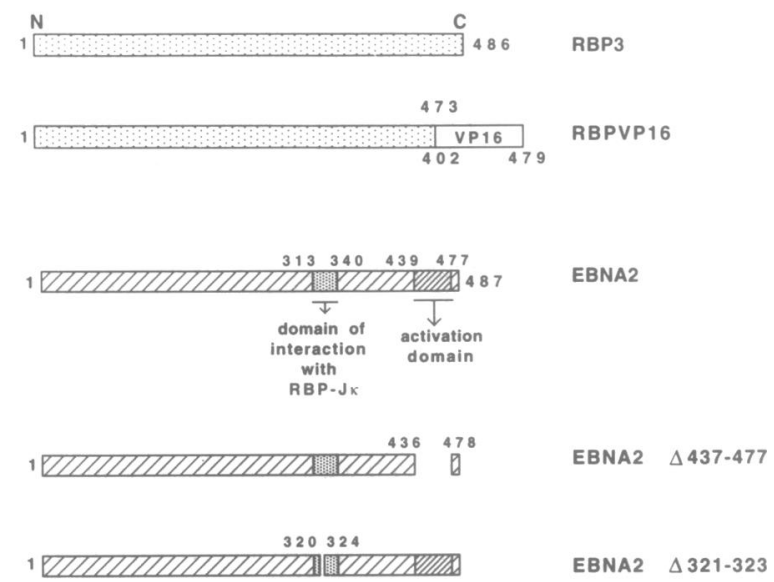

EBNA2 $\triangle 437-477$ EBNA2 $\triangle 321-323$

B

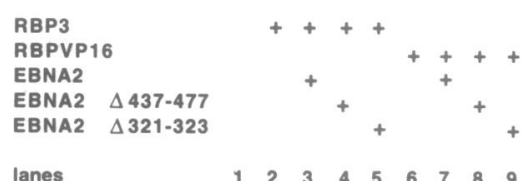

lanes

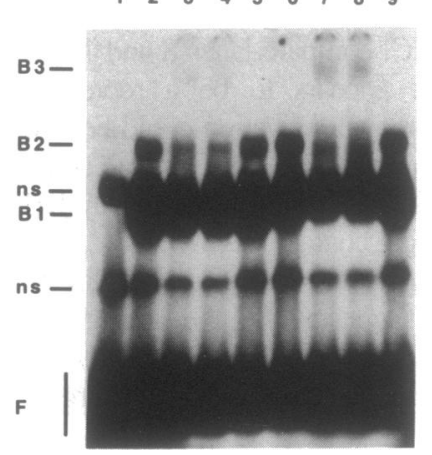

Figure 2. RBP-VP16 fusion protein and wild-type RBP3 both bind DNA and interact with EBNA2 and EBNA2 $4437-477$, but not with EBNA2 $321-323$, in vitro. (A) Schematic representation of the proteins used in the EMSA experiments. In EBNA2 $4437-477$ the activation domain of EBNA2 is deleted. In EBNA2 $\triangle 321-323$ the two tryptophans (322 and 323) absolutely necessary for EBNA2 interaction with RBP-JK have been deleted. (B) EMSA were performed with in vitro-translated RBP3 (lanes 2-5) or RBP-VP16 (lanes 6-9) and a radiolabeled double-stranded probe (TP) containing two binding sites for RBP-J-K. 5'-CTCGCGACTCGTGGGAAAATGGGCGGAAGGGCACCGTGGGAAAA TAGTTCCAGG-3'. In vitro-translated EBNA2, EBNA2 $437-477$ or EBNA2 $\Delta 321-323$. The bands are indicated as follows. F, free probe; ns, non-specific complex; $\mathrm{B} 1$ and $\mathrm{B} 2$, complexes formed by binding of one or two RBP3 molecules respectively; B3, EBNA2 or EBNA2 derivative supershifted complexes.

\section{Repression by RBP-JK involves a co-repressor}

Although DNA-bound RBP-VP16 activated transcription, it was possible that this activation was not optimal, but partially repressed either by endogenous RBP-JK bound to adjacent sites or by the RBP3 moiety in RBP-VP16. In either case this repression could be exerted either directly or through a specific co-effector(s). In order to examine the existence of such a co-effector we made use of RBP3 derivatives deficient in their ability to bind DNA. Such proteins could be expected to trap and

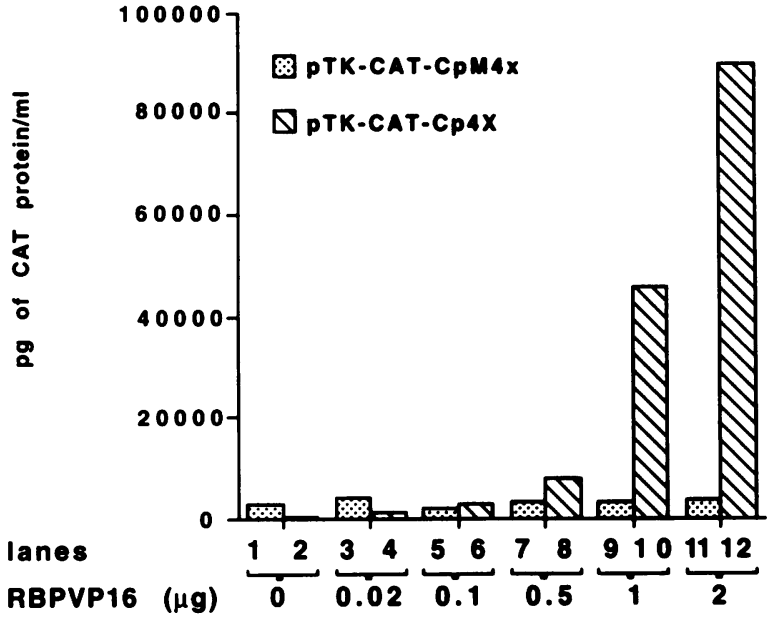

Figure 3. RBP-VP16 activates transcription from pTK-CAT-Cp4x. pTK-CATCpM4x (dotted columns) or pTK-CAT-Cp4x (hatched columns) reporter plasmids $(10 \mu \mathrm{g})$ were transfected into HeLa cells with increasing amounts of RBP-VP16 expression vector. Promoter activity was assayed as before.

titrate the potential co-effector. We thus generated site-directed RBP3 mutants (Fig. 4A) and expressed them in vitro using a coupled transcription/translation system (Fig. 4B). As evaluated by EMSA, four mutants (RBP KR50SL, RBP RL178GI, RBP HK269SL and RBP WT314SL) had strongly decreased DNA binding activity compared with wild-type RBP3 protein (Fig. 4C). RBP KR50SL and RBP RL178GI were selected for further study, since their binding activity was the lowest. When expressed in HeLa cells these proteins were present in comparable amounts and were correctly imported into the nucleus (data not shown).

We next evaluated the effect of the two mutant proteins RBP KR50SL and RBP RL178GI on RBP-VP16-activated transcription. For this we used the reporter plasmid pTATA-CAT-Cp4x (Fig. 5A), consisting of the CAT gene placed under the control of four RBP-JK binding sites cloned upstream of the TK TATA box. Upon transfection of pTATA-CAT-Cp4x into HeLa cells transcription of the CAT gene was inefficient (Fig. 5B, lane 1). However, it was activated by RBP-VP16 (Fig. 5B, lane 2), whereas RBP KR50SL and RBP RL178GI gave no activation (Fig. 5B, lanes 3 and 4 respectively). Interestingly, when RBP KR50SL (Fig. 5B, lanes 6-8) or RBP RL178GI (Fig. 5B, lanes 9-11) were co-expressed with RBP-VP16 transcription of the $C A T$ gene was increased as compared with that induced by RBP-VP16 alone (Fig. 4B, lane 2). It should be noted that the level of $C A T$ expression was directly proportional to the amount of RBP KR50SL or RBP RL178GI expressing plasmid transfected. Taken together these results are consistent with the hypothesis that a co-repressor(s) binds to RBP-JK and can be sequestered by RBP3 DNA binding-defective mutants. To further evaluate this hypothesis we introduced a second mutation in protein RBP KR50SL that has previously been shown to strongly impair repression mediated by a Gal4-RBP-JK fusion (35). This doubly mutated protein, called RBP KR50SL/EEF219AAA (Fig. 4A), did not bind DNA in vitro and upon transfection into HeLa cells had no effect on RBP-VP16-mediated transcription (Fig. 5B, lanes 12-14). Thus the negative effect of the EEF219AAA mutation on the capacity to titrate the potential co-repressor correlates with the negative effect of this mutation 
A

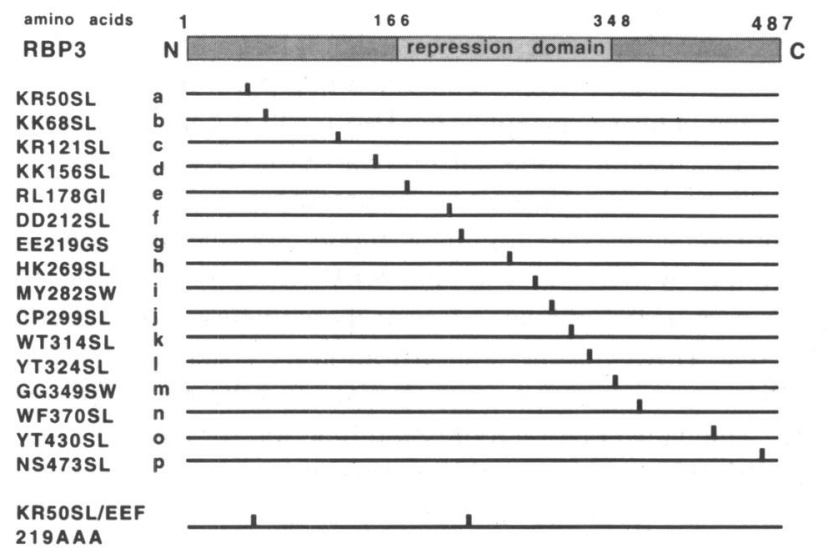

B

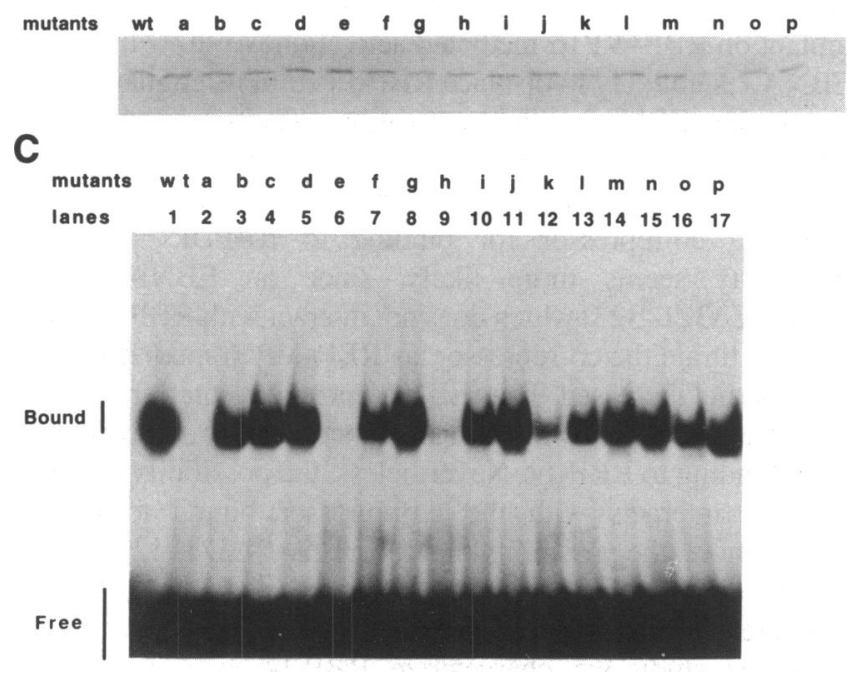

Figure 4. Characterization of DNA binding-defective RBP-JK mutant proteins. (A) Schematic representation of the RBP-JK isoform RBP3 and the different mutant proteins. The location of the mutated residues is indicated in the diagrams. For each mutant the first two letters refer to the amino acids mutated. The number that follows refers to their position in the primary sequence and the last two letters indicate the changes made. (B) Quantification of the in vitro-translated RBP3 mutants by SDS-PAGE. The proteins are $\left[{ }^{14} \mathrm{C}\right]$ Leu labeled. (C) EMSA was performed with the double-stranded radiolabeled $\mathrm{Cp}$ probe 5'-TCGAGGTGTAAACACGCCGTGGGAAAAAATTTATGTCGA-3' using equivalent amounts of the in vitro-translated mutant proteins.

on the repression activity of Gal4-RBP-JK as described by Hsieh et al. (35). These results strengthen the hypothesis that a co-repressor mediates RBP-JK-induced repression.

\section{EBNA2 counteracts RBP-JK-mediated repression neither by destabilizing RBP-JK binding nor by directly interacting with the co-repressor}

As shown above, RBP-JK-mediated repression occurs via a co-repressor(s). EBNA2 could impair this repression by destabilizing RBP-JK binding to DNA, as suggested by in vitro experiments (20). Alternatively, EBNA2 could interfere directly with the co-repressor function or with its binding to RBP-JK. In order to evaluate these possibilities we generated an EBNA2

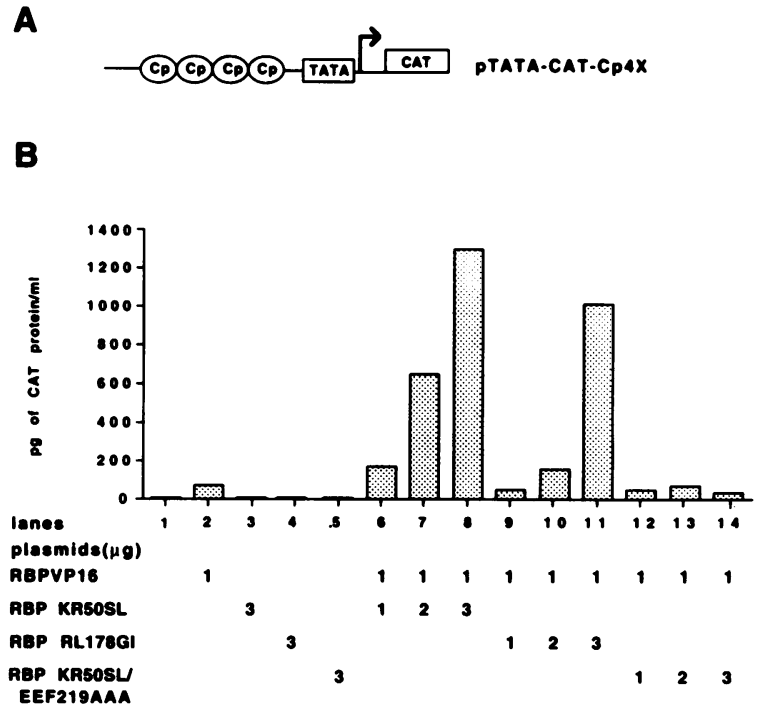

Figure 5. Titration of RBP-JK-associated co-repressor by DNA binding-defective RBP3 mutants. (A) Diagram of the reporter plasmid pTATA-CAT-Cp4x used. (B) HeLa cells were transfected with $10 \mu \mathrm{g}$ pTATA-CAT-Cp4x reporter plasmid and various combinations of expression plasmids as indicated in the lower part of the panel. The amounts of transfected expression plasmids coding for RBP-VP16, RBP KR50SL, RBP RL178GI and RBP KR50SL EEF219AAA are indicated in $\mu \mathrm{g}$. The transcriptional activity was assayed by CAT-ELISA as described above.

mutant (EBNA2 $\triangle 437-477$ ) (Fig. 2A) with its activation domain deleted (17,37). As expected, when expressed in HeLa cells EBNA2 $\triangle 437-477$ alone did not detectably activate transcription from pTATA-CAT-Cp4x (Fig. 6, lane 3). However, although EBNA2 $437-477$ destabilized RBP3 (or RBP-VP16) DNA binding in vitro in an EMSA (Fig. 2B, lanes 4 and 8), this destabilizing effect was not observed upon co-transfection of RBP-VP16 with EBNA2 $437-477$ in HeLa cells. Indeed, transcriptional activation from the pTATA-CAT-Cp4x reporter plasmid by RBP-VP16 (Fig. 6, lane 2) not only was not decreased by co-expression of EBNA2 $\triangle 437-477$, but was in fact strongly enhanced (up to 100 times) when EBNA2 $\Delta 437-477$ was co-expressed with RBP-VP16 (Fig. 6, lanes 5-8). This enhancement was dependant on a physical interaction between EBNA2 and RBP-JK, since the EBNA2 mutant (EBNA2 $321-323$, Fig. 2A) which no longer interacted in vitro with RBP3 (or RBP-VP16) (Fig. 2B, lane 5 and 9) did not significantly affect RBP-VP16 activation (Fig. 6, lanes 9-12). Furthermore, this latter result also suggests that EBNA2 does not directly contact the co-repressor. Indeed, in the case of a direct contact between EBNA2 and the co-repressor a titration of this co-repressor by the RBP binding-deficient mutant EBNA2 $\triangle 321-323$ would be expected and we should observe an inhibition of repression. However, our results show that the EBNA2 $2321-323$ mutant does not have this effect.

Taken together these results suggest that EBNA2 does not destabilize RBP-JK DNA binding in vivo, but, by interacting with RBP-JK, impairs RBP-JK-mediated repression. 


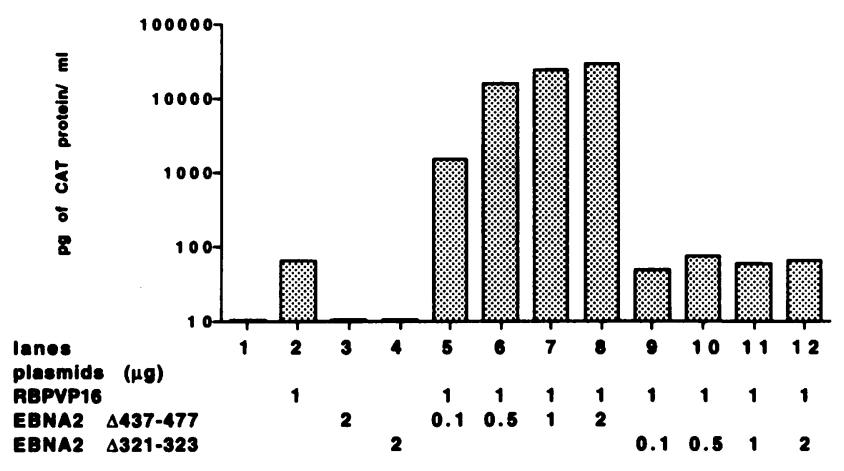

Figure 6. EBNA2 counteracts RBP-JK-mediated repression neither by destabilizing RBP-JK binding to DNA nor by interacting directly with the co-repressor. pTATA-CAT-Cp4x reporter plasmid $(10 \mu \mathrm{g})$ was transfected into HeLa cells with either RBP-VP16, EBNA2 $437-477$ or EBNA2 $321-323$ expression vector alone (lanes 2,3 and 4 respectively) or with $1 \mu \mathrm{g}$ RBP-VP16 expression vector and increasing amounts of EBNA2 $\Delta 437-477$ or EBNA2 $\Delta 321-323$ expression vector (lanes 5-8 and 9-12 respectively). The amounts of expression vector transfected are indicated in $\mu \mathrm{g}$.

\section{DISCUSSION}

One of the mechanisms by which EBNA2 activates transcription is through interaction with the cellular DNA binding protein RBP-JK. It has been proposed (35) that by directly contacting RBP-JK, EBNA2 could both mask a repression domain in RBP-JK (derepression) and provide an activation domain (recruitment). This model derives from results obtained using a fusion protein made between RBP-JK and the Gal4 DNA binding domain. This approach allowed identification of a transcriptional repression domain in RBP-JK. Furthermore, EBNA2, by directly interacting with Gal4-RBP-JK, both alleviated this repressing activity and activated transcription (35). Here we show that this model can be extended to RBP-JK bound to its cognate DNA binding sites and that the two steps, derepression and recruitment, can be uncoupled. We also show that RBP-JK-mediated repression requires a co-repressor.

Our results show that a fusion between RBP-JK and the VP16 activation domain (RBP-VP16) is able to activate transcription from a promoter containing RBP-JK binding sites. Using this construct we could mimic the recruitment of an activation domain by RBP-JK without concomitant masking of the RBP-JK repression domain. Thus it is possible to counterbalance RBPJK-mediated repression without blocking activity of the repression domain. However, this RBP-VP16-mediated activation was not optimal, as revealed by the experiments using RBP3 DNA binding-deficient derivatives or EBNA2 with its activation domain deleted. This could be due either to repression by the RBP3 moiety of RBP-VP16 or by endogenous RBP-JK bound to adjacent sites.

Although Hsieh et al. mapped a repression domain in RBP-JK (35), the question of whether this domain functions directly or through a co-repressor(s) has remained unanswered. Here we provide evidence that $\mathrm{RBP}-\mathrm{JK}$-mediated repression requires another cellular protein present in limiting amounts in the cell. By co-expressing DNA binding-defective RBP-JK mutants with RBP-VP16 we observed an increase in RBP-VP16-mediated activation consistent with titration of a co-repressor. We also analyzed in our system the effect of a point mutation, previously described by Hsieh et al, that abolishes Gal4-RBPJK-mediated repression (35). Our results show that a DNA binding-defective RBP-JK derivative which contained this point mutation did not titrate the putative co-repressor. This result is consistent with the hypothesis that the repression domain defined by Hsieh et al. (35) is implicated in binding of a co-repressor.

Until recently the dominant model for the mechanism of activation by EBNA2 was that RBP-JK targets EBNA2 to its DNA responsive elements where its activation domain can be effective. However, the demonstration by Hsieh et al. (35) that RBP-JK contains a repression domain and the results presented here, which also favor a repression function for RBP-JK, suggest that EBNA2 could inhibit this repression. One of the mechanisms by which this could occur is by displacing DNA-bound RBP-JK. This possibility is supported by the strong destabilizing effect of EBNA2 on RBP-JK DNA binding observed in vitro. Here, by analyzing the effect of an EBNA2 activation domain deletion mutant on RBP-VP16-mediated activation we show that in vivo EBNA2 is unlikely to displace RBP-JK from its cognate binding site. In fact, EBNA2 with its activation domain deleted increased RBP-VP16-mediated activation. Therefore, EBNA2 either directly contacts and inactivates the co-repressor or competes with the co-repressor for binding to RBP-JK. This latter possibility seems more likely, since an EBNA2 mutant (EBNA2 $4321-323$ ) which does not interact with RBP-JK did not seem to titrate the co-repressor, as RBP-VP16-mediated activation was not increased. Thus EBNA2 probably interferes with the co-repressor, either by blocking its function or by competing with it for binding to RBP-JK. Nevertheless, the possibility exists that EBNA2 interacts with the co-repressor bound to RBP-JK. Furthermore, the possibility that the EBNA2 $\Delta 321-323$ mutation affects the putative interaction domain of EBNA2 with the co-repressor cannot be excluded.

In this study we characterize RBP-JK as a repressor in mammalian cells, in agreement with Dou et al. (34) and Hsieh et al. (35), but RBP-JK and its homologous Drosophila protein $\mathrm{Su}(\mathrm{H})$ have been described as transcriptional activators $(28)$ in Drosophila S2 cells. However, these latter results could be explained by a lack of co-repressor in this particular cell line and/or by the presence of an EBNA2-like cellular activator.

In conclusion, transactivation by EBNA2 is more likely due to interference with the function of a co-repressor recruited by RBP-JK than destabilization of RBP-JK binding to DNA. Furthermore, EBNA2 recruited to the promoter by RBP-JK provides an activation domain which could then recruit components of the basal transcription machinery to the promoter. Further regulation of the function(s) of RBP-JK could be achieved by interaction with additional factors. In B cells latently infected with EBV it has been shown that EBNA2-induced activation can be inhibited by the EBNA3 proteins (38-41). In particular, EBNA3c has been shown to directly interact with RBP-JK, thus destabilizing DNA-RBP-JK complexes in vitro (38). In Drosophila $\mathrm{S} 2$ cells interaction between Hairless and $\mathrm{Su}(\mathrm{H})$ (the RBP-JK Drosophila homolog) leads to inhibition of $\mathrm{Su}(\mathrm{H})$-mediated activation, probably by destabilizing the association of RBP-JK with DNA (28). In addition, in the absence of the Notch ligand the activity of $\mathrm{Su}(\mathrm{H})$ would be controlled via cytoplasmic retention by the Notch membrane receptor protein (42). 
The interaction of RBP-JK with a number of different cellular and viral proteins provides a framework for analyzing the function of RBP-JK. Study of the precise protein-protein interactions and characterization of the RBP-JK domain(s) involved in contact with these various proteins will be particularly important for understanding RBP-JK-mediated transcriptional regulation. Moreover, the identification and characterization of other RBP-JK partners, among them the potential co-repressor discussed here, will be of primary importance to shed further light on RBP-JK function.

\section{ACKNOWLEDGEMENTS}

We wish to thank Conrad B.Bluink for reading the manuscript. This work was financially supported by INSERM, by the Association pour la Recherche sur le Cancer (ARC 6810) and by FNCLCC.

\section{REFERENCES}

1 Liebowitz,D. and Kieff,E. (1993) In Roizman,B., Whitley,R.J. and Lopez,C.(eds), The Human Herpes Viruses. Raven Press, New York, NY, pp. 107-172.

2 Cohen,J.F., Wang,F., Mannick,J. and Kieff,E. (1989) Proc. Natl. Acad. Sci. USA, 86, 9558-9562.

3 Hammerschmidt,W. and Sugden,B. (1989) Nature, 340, 393-397.

4 Sinclair,A.J., Palmero,I., Peters,G. and Farrell,P.J. (1994) EMBO J., 13, 3321-3328.

5 Kempkes,B., Spitkovsky,D., Jansen-Dürr,P., Ellwart,J.W., Kremmer,E., Delecluse,H.-J., Rottenberger,C., Bornkamm,G.W. and Hammerschmidt,W. (1995) EMBO J., 14, 88-96.

6 Calender,A., Billaud,M., Aubry,J.-P., Banchereau,J., Vuillaume,M. and Lenoir,G.M. (1987) Proc. Natl. Acad. Sci. USA, 84, 8060-8064.

7 Cordier,M., Calender,A., Billaud,M., Zimber,U., Rousselet,G., Pavlish,O., Banchereau,J., Tursz,T., Bornkamm,G. and Lenoir,G.M. (1990) J. Virol., 64, 1002-1013.

8 Knuston,J.C. (1990) J. Virol., 64, 2530-2536.

9 Wang,F., Gregory,C., Sample,C., Rowe,M., Liebowitz,D., Murray,R., Rickinson,A. and Kieff,E. (1990) J. Virol., 64, 2309-2318.

10 Wang,F., Tsang,S.-F., Kurilla,M.G., Cohen,J.I. and Kieff,E. (1990) J. Virol., 64, 3407-3416.

11 Zimber-Strobl,U., Suentzenich,K.-O., Laux,G., Eick,D., Cordier,M., Calender,A., Billaud,M., Lenoir,G.M. and Bornkamm,G.W. (1991) J. Virol., 65, 415-423.

12 Abbot,S.D., Rowe,M., Cadwallader,K., Ricksten,A., Gordon,J., Wang,F., Rymo,L. and Rickinson,A.B. (1990) J. Virol., 64, 2126-2134.

13 Fahraeus,R., Jansson,A., Ricksten,A., Sjôblom,A. and Rymo,L. (1990) Proc. Natl. Acad. Sci. USA, 87, 7390-7394.
14 Sung,N.S., Kenney,S., Gutsch,D. and Pagano,J.S. (1991) J. Virol., 65, 2164-2169.

15 Rooney,C.M., Brimmell,M., Buschle,M., Allan,G., Farrell,P.J. and Kolman,J.L. (1992) J. Virol., 66, 496-504.

16 Woisetchlaeger,M., Yandava,C.N., Furmanski,L.A., Strominger,J.L. and Speck,S. (1990) Proc. Natl. Acad. Sci. USA, 87, 1725-1729.

17 Cohen,J.I., Wang,F. and Kieff,E. (1991) J. Virol., 65, 2545-2554.

18 Zimber-Strobl,U., Kremmer,E., GrSsser,F., Marschall,G., Laux,G. and Bornkamm,G.W. (1993) EMBO J., 12, 167-175.

19 Ling,P.D., Rawlins,D.R. and Hayward,S.D. (1993) Proc. Natl. Acad. Sci. USA, 90, 9237-9241.

20 Waltzer,L., Logeat,F., Brou,C., Israel,A., Sergeant,A. and Manet,E. (1994) EMBO J., 13, 5633-5638.

21 Henkel,T., Ling,P.D., Hayward,L.S.D. and Peterson,M.G. (1994) Science, 265, 92-95.

22 Grossman,S.R., Johannsen,E., Tong,X., Yalamanchili,R. and Kieff,E. (1994) Proc. Natl. Acad. Sci. USA, 91, 7568-7572.

23 Yalamanchili,R., Tong,X., Grossman,S., Johannsen,E., Mosialos,G. and Kieff,E. (1994) Virology, 204, 634-641.

24 Zimber-Strobl,U., Strobl,L.J., Meitinger,C., Hinrichs,R., Sakai,T., Furukawa,T., Honjo,T. and Bornkamm,G. (1994) EMBO J., 13, 4973-4982.

25 Hamaguchi,Y., Matsumani,N., Yamamoto,Y. and Honjo,T. (1989) Nucleic Acids Res., 17, 9015-9026.

26 Matsumani,N., Hamaguchi,Y., Yamamoto,Y., Kuze,K., Kangawa,K., Matsuo,H., Kawaichi,M. and Honjo,T. (1989) Nature, 342, 934-937.

27 Furukawa,T., Kawaichi,M., Matsunami,N., Ryo,H., Nishida,Y. and Honjo,T. (1991) J. Biol. Chem., 266, 23334-23340.

28 Brou,C., Logeat,F., Lecourtois,M., Vandekerckhove,J., Kourilsky,P., Schweisguth,F. and Israel,A. (1994) Genes Dev., 8, 2491-2503.

29 Amakawa,R., Jing,W., Ozawa,K., Matsunami,N., Hamaguchi,Y., Matsumada,F., Kawaichi,M. and Honjo,T. (1993) Genomics, 17, 306-315.

30 Tun,T., Hamaguchi,Y., Matsunami,N., Furukawa,T., Honjo,T. and Kawaichi,M. (1994) Nucleic Acids Res., 22, 965-971.

31 Furukawa,T., Maruyama,S., Kawaichi,M. and Honjo,T. (1992) Cell, 69, 1191-1197.

32 Schweisguth,F. and Posakony,J.W. (1992) Cell, 69, 1199-1212.

33 Schweisguth,F. and Posakony,J.W. (1994) Development, 120, 1433-1441.

34 Dou,S., Zeng,X., Cortes,P., Erdjument-Bromage,H., Temps,P., Honjo,T. and Vales,L.D. (1994) Mol. Cell. Biol., 14, 3310-3319.

35 Hsieh,J.J.-D. and Hayward,S.D. (1995) Science, 238, 560-563.

36 Ace,C.I., Dalrymple,M.A., Ramsay,F.H., Preston,V.G. and Preston,C.M. (1988) J. Gen. Virol., 69, 2595-2605.

37 Cohen,J.I. (1992) Proc. Natl. Acad. Sci. USA, 89, 8030-8034.

38 Robertson,E.S., Grossman,S., Johannsen,E., Miller,C., Lin,J., Tomkinson,B. and Kieff,E. (1995) J. Virol., 69, 3108-3116.

39 Le Roux,A., Kerdiles,B., Walls,D., Dedieu,J.F. and Perricaudet,M. (1994) Virology, 205, 596-602.

40 Marshall,D. and Sample,C. (1995) J. Virol., 69, 3624-3630.

41 Sample,C. and Parker,B. (1994) Virology, 205, 534-539.

42 Fortini,M.E. and Artavanis-Tsakonas,S. (1994) Cell, 79, 273-282. 